\title{
SOLUTION OF 3D CONTACT PROBLEMS USING SPLINE INTERPOLATION
}

\author{
Attilla Baksa And Isván PÁCZelt \\ University of Miskolc \\ Department of Applied Mechanics, HUNGARY \\ mechab@uni-miskolc.hu, mechpacz@uni-miskolc.hu \\ TAMÁs SzABÓ \\ Széchenyi István University, Győr, \\ Department of Applied Mechanics, HUNGARY \\ tom.tolcsva@gmail.com
}

[Received: March 15, 2009]

\begin{abstract}
A three-dimensional elastic contact problem is examined. It is assumed that the displacements and deformations are small and the supposed zone of the contact domain must be single-connected, and surrounded by a smooth closed curve. The closed curve is interpolated by a single closed spline. For numerical tests three-dimensional $p$-extension finite elements are used. To take the conditions of contact and separation into account the penalty method is applied. Numerical examples presented here show the effect of positioning 3D $p$-version elements along the contact-separation and/or stick-and-slip borders and the influence of the friction coefficient, respectively.
\end{abstract}

Mathematical Subject Classification: 37J05, 76M10, 65D05, 65D10

Keywords: Contact problem, frictional contact, p-extension FEM, B-spline, interpolation with a closed spline, contact element positioning

\section{InTRODUCTION}

The modern history of contact mechanics might be said to have begun in 1882 with the publication by Hertz of his classic paper [1]. The classical Signorini problem [2] formulated in 1933 leading to the developement of variational inequalities for the contact problem. These methods have been developed within several decades. After some analytical approached published by Muskelishvili [3] or Galin [4] there are numerous researchers who also worked on these fields e.g. Goldsmith [5], Fichera [6] or Kikuchi and Oden [7] and see some books for historical remarks in Johnson [8, in Kalker [9] or in Wriggers [10].

The finite element method (FEM) developed together with the growing power of modern computers. Turner and his colleagues in [11] have taken the first attempts to solve structural problems using finite elements in the late fifties. A further decade 
must have gone by before the first papers, in which contact problems solved by FEM, appeared [12].

FEM can be classified into three main groups with respect to the type of refinement: $h-, p$-, and $h p$-extension. In the case of $h$-extension the size of the elements are decreased, while the $p$-extension increases only the polynomial degree of shape functions in order to give more accurate results. In recent years the interest in high order finite elements has been increased.

It was proved by Szabó and Babuška [13 that even in the case of singularities in the exact solution, the $p$-extension in combination with a proper mesh ensure an exponential rate of convergence in energy norm. If the mesh is aligned to points or lines of singularity, from practical point of view an adequate accuracy can be obtained. It was examined by Páczelt et al. in [14, 15] for two-dimensional examples of normal and frictional contact problems.

As the problem investigated in this paper is restricted to contact between convex bodies bounded by $C^{2}$ surfaces, and assuming small displacements and deformations, in this case of $3 \mathrm{D}$ contact problems the resulting contact domain is not a line, like in $2 \mathrm{D}$, but a single-connected surface, which is bordered by a smooth closed curve. To ensure precise numerical results for 3D contact problems using $p$-extension elements, the determination of the contact zone or its three-dimensional contour is the key question of our investigation.

Article 16] examines the problems of elasto-plasticity with adaptive p-FEM. The present paper describes also an adaptive mesh modification technique which uses an interpolating spline curve. For an excellent and comprehensive treatment of Bsplines from the numerical analyst's point of view, de Boor has served as the standard reference for close to 30 years [17. A good introduction to Non-Uniform Rational Bsplines, referred to as NURBS, can be found in [18. A key advantage of using NURBS is the ability to represent conic sections and quadratic surfaces, such as ellipse, circle and cones [19. Moreover, the free-form curves and surfaces are all a special case of the NURBS since the constant function 1 is a perfectly respectable spline, and can be used as the denominator in a rational representation.

During the last ten years, research activities have been focused on adaptive techniques that provide a discretization which is accurate and reliable. Adaptive techniques rely on indicators or estimators, which are able to predict the error of the approximated solution [13.

In the present article a solid and a linear elastic body's contact problem will be examined with adaptive $p$-extension FEM. Numerical examples show the convergence of the solution and the connection between the polynomial degree of shape functions and the relative error of the numerical results.

\section{The PRoblem of ElASTiCity}

The considered mechanical system consists of two three-dimensional homogenous and isotropic elastic bodies. Only the mechanical interactions between them will be 
examined. It is supposed that the displacements and deformations are small, and deformations evolving from external loads are only elastic, i.e. the task to be solved is in the frame of linear elasticity.

The examined contacting bodies (shown in Figure1) have the volume $V^{\alpha}$ and outer boundary $S^{\alpha}$, in which $\alpha=1,2$ marks the referred particle. The outer boundary is separeted into three parts: on surface $S_{u}^{\alpha}$ the displacements $\boldsymbol{u}_{0}^{\alpha}$ are given; on surface $S_{p}^{\alpha}$ the surface traction $\widetilde{\boldsymbol{p}}^{\alpha}$ is prescribed; while the surface $S_{c}^{\alpha}$ is the supposed zone of contact.

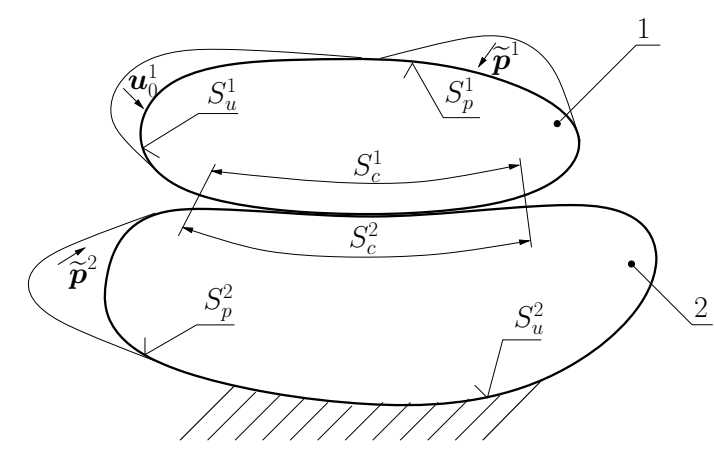

Figure 1. The examined contacting bodies

According to problem of elasticity the equilibrium equation is

$$
\boldsymbol{\sigma}^{\alpha} \cdot \nabla+\boldsymbol{b}^{\alpha}=\mathbf{0} \quad \forall \boldsymbol{r}^{\alpha} \in V^{\alpha}
$$

in which $\boldsymbol{b}^{\alpha}$ is the body force, $\boldsymbol{\sigma}^{\alpha}=\boldsymbol{\sigma}^{\alpha}\left(\boldsymbol{r}^{\alpha}\right)$ is the stress tensor, $\nabla$ is the nabla operator. Strains are calculated as

$$
\varepsilon^{\alpha}=\frac{1}{2}\left(\boldsymbol{u}^{\alpha} \circ \nabla+\nabla \circ \boldsymbol{u}^{\alpha}\right) \quad \forall \boldsymbol{r}^{\alpha} \in V^{\alpha},
$$

where $\varepsilon^{\alpha}=\varepsilon^{\alpha}\left(\boldsymbol{r}^{\alpha}\right)$ is the strain tensor. The constitutive equation is

$$
\boldsymbol{\sigma}^{\alpha}=\boldsymbol{D}^{\alpha} \cdots \varepsilon^{\alpha} \quad \forall \boldsymbol{r}^{\alpha} \in V^{\alpha},
$$

in which $\boldsymbol{D}^{\alpha}$ is the fourth order elasticity tensor.

In equation (2.1) scalar product is denoted by a dot, double dots indicate the scalar product between two tensors in equation (2.3), and in equation 2.2 the circle denotes the dyadic (tensorial) product according to Lurje [20].

The natural and essential boundary conditions are defined as

$$
\boldsymbol{\sigma}^{\alpha} \cdot \boldsymbol{n}^{\alpha}=\widetilde{\boldsymbol{p}}^{\alpha} \quad \forall \boldsymbol{r}^{\alpha} \in S_{p}^{\alpha}, \quad \boldsymbol{u}^{\alpha}=\boldsymbol{u}_{0}^{\alpha} \quad \forall \boldsymbol{r}^{\alpha} \in S_{u}^{\alpha} .
$$

The above equations 2.1 -2.4 must be complemented with the conditions of contact-separation which are valid on $S_{c}^{\alpha}$ in order to fullfill all the requirements of the contact problem of linear elasticity. 
2.1. Contact and separation. The outer normal of body $\alpha$ is denoted by $\boldsymbol{n}^{\alpha}$. In the domain of the supposed zone of contact $S_{c}^{\alpha}$ the contact normal is defined as $\boldsymbol{n}_{c}=-\boldsymbol{n}^{2}=\boldsymbol{n}^{1}$. Before loading, the distance $h$ between the bodies is measured along the normal $\boldsymbol{n}_{c} . h$ is decreased to the distance between the bodies after deformation is

$$
d=d(\boldsymbol{u})=u_{n}^{2}-u_{n}^{1}+h \quad \forall \boldsymbol{r}^{\alpha} \in S_{c}^{\alpha},
$$

which is shown in Figure 2. The normal components of the displacements are calculated as $u_{n}^{\alpha}=\boldsymbol{u}^{\alpha} \cdot \boldsymbol{n}_{c}$.
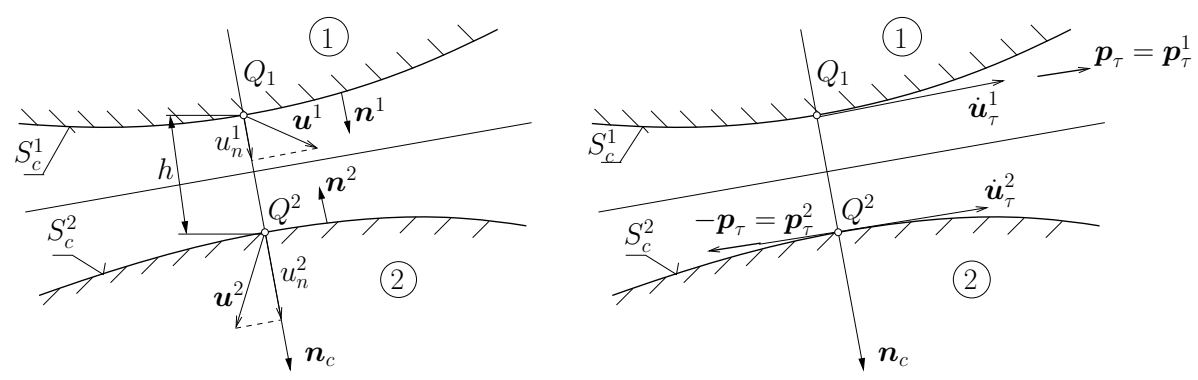

Figure 2. Contact and separation of two bodies

The normal components of the stresses on domain $S_{c}^{\alpha}$ can be determined as follows

$$
\sigma_{n}^{\alpha}=\boldsymbol{n}^{\alpha} \cdot \boldsymbol{\sigma}^{\alpha} \cdot \boldsymbol{n}^{\alpha} \quad \forall \boldsymbol{r}^{\alpha} \in S_{c}^{\alpha} .
$$

The contact pressure $p_{n}$ is defined through the normal stress

$$
p_{n}=-\sigma_{n}^{1}=-\sigma_{n}^{2} \quad \forall \boldsymbol{r}^{\alpha} \in S_{c}^{\alpha},
$$

and the tangential stress vectors

$$
\boldsymbol{p}_{\tau}^{\alpha}=\boldsymbol{\sigma}^{\alpha} \cdot \boldsymbol{n}^{\alpha}-\sigma_{n}^{\alpha} \boldsymbol{n}^{\alpha}, \quad \boldsymbol{p}_{\tau}=\boldsymbol{p}_{\tau}^{1}=-\boldsymbol{p}_{\tau}^{2} .
$$

Summarising the statements written above the following conditions for contact and separation can be achieved.

(a) conditions of contact

$$
d=0, \quad p_{n}>0 \quad \forall \boldsymbol{r} \in \Omega_{p},
$$

(b) conditions of separation

$$
d>0, \quad p_{n}=0 \quad \forall \boldsymbol{r} \in \Omega_{0},
$$

(c) complementary conditions

$$
d \geq 0, \quad p_{n} \geq 0, \quad p_{n} d=0 \quad \forall \boldsymbol{r} \in S_{c}=\Omega_{p} \cup \Omega_{0} .
$$

The formulas $2.9 \mathrm{a}-2.9 \mathrm{c})$, called Hertz-Signonirini-Moreau conditions, give the basis of the solution of frictionless normal contact problems. 
2.2. Stick and slip. Tangential stresses in the case of Coulomb-type friction are

$$
\left\|\boldsymbol{p}_{\tau}^{\alpha}\right\|=\left\|\boldsymbol{\sigma}^{\alpha} \cdot \boldsymbol{n}^{\alpha}-\sigma_{n}^{\alpha} \boldsymbol{n}^{\alpha}\right\| \leq \mu p_{n} .
$$

The tangential relative velocity is $\dot{\boldsymbol{u}}_{\tau}=\dot{\boldsymbol{u}}_{\tau}^{2}-\dot{\boldsymbol{u}}_{\tau}^{1}$. The effects of friction are treated analogously to plasticity according to Michalowski and Mróz [21, i.e.

$$
\begin{gathered}
\dot{\boldsymbol{u}}_{\tau}=\dot{\boldsymbol{u}}_{\tau}^{(a d h)}+\dot{\boldsymbol{u}}_{\tau}^{(\text {slip })} \\
\dot{\boldsymbol{u}}_{\tau}^{(a d h)}=\frac{\dot{\boldsymbol{p}}_{\tau}}{c_{\tau}}, \quad \dot{\boldsymbol{u}}_{\tau}^{(s l i p)}=\dot{\xi} \frac{\partial \phi}{\partial \boldsymbol{p}_{\tau}}, \quad \phi=\left\|\boldsymbol{p}_{\tau}\right\|-\mu p_{n} \leq 0,
\end{gathered}
$$

where $c_{\tau}$ is the stiffness of connections at the interface and $\phi$ is the slip surface, $\dot{\xi} \geq 0$.

(a) With friction $\left(\Omega_{p}=\Omega_{p}^{a}+\Omega_{p}^{s}\right)$

- on stick zone:

$$
\dot{\boldsymbol{u}}_{\tau}(\boldsymbol{r})=\dot{\boldsymbol{u}}_{\tau}^{2}(\boldsymbol{r})-\dot{\boldsymbol{u}}_{\tau}^{1}(\boldsymbol{r})=0 \quad \text { and } \quad\left\|\boldsymbol{p}_{\tau}^{\alpha}\right\| \leq \mu p_{n} \quad \boldsymbol{r} \in \Omega_{p}^{a},
$$

- on slip zone:

$$
\dot{\boldsymbol{u}}_{\tau}(\boldsymbol{r}) \neq 0, \quad \text { and } \quad \boldsymbol{p}_{\tau}=\mu p_{n} \frac{\dot{\boldsymbol{u}}_{\tau}(\boldsymbol{r})}{\left\|\dot{\boldsymbol{u}}_{\tau}(\boldsymbol{r})\right\|} \quad \boldsymbol{r} \in \Omega_{p}^{s} .
$$

(b) Without friction

$$
\boldsymbol{p}_{\tau}^{\alpha}=\mathbf{0} .
$$

Using the plasticity analogy the conditions for adhesion and slip are as follows

- in the adhesion (stick) zone

$$
\phi<0, \quad \dot{\xi}=0
$$

- in the slip zone

$$
\phi=0, \quad \dot{\xi}>0
$$

which ensures that $\phi \dot{\xi}=0$.

Therefore the tangential stress can be expressed as

$$
\dot{\boldsymbol{p}}_{\tau}=c_{\tau} \dot{\boldsymbol{u}}_{\tau}-c_{\tau} \dot{\boldsymbol{u}}_{\tau}^{(s l i p)}=c_{\tau} \dot{\boldsymbol{u}}_{\tau}-c_{\tau} \dot{\xi} \frac{\partial \phi}{\partial \boldsymbol{p}_{\tau}} .
$$

The quasi-static problem is treated by the backward Euler time-discretization technique. So the time integration of 2.18) on the interval from $t$ to $t+\Delta t$ gives

$$
{ }^{t+\Delta t} \boldsymbol{p}_{\tau}=\boldsymbol{p}_{\tau}^{*}-c_{\tau} \Delta \xi\left[\frac{\partial \phi}{\partial \boldsymbol{p}_{\tau}}\right]^{t+\Delta t}=\boldsymbol{p}_{\tau}^{*}-c_{\tau} \Delta \xi\left[\frac{\partial \phi}{\partial \boldsymbol{p}_{\tau}}\right]^{*}=\boldsymbol{p}_{\tau}^{*}-\boldsymbol{p}_{\tau, m_{0}}^{*}
$$

where the predicted shear stress is

$$
\boldsymbol{p}_{\tau}^{*}={ }^{t} \boldsymbol{p}_{\tau}+c_{\tau}\left({ }^{t+\Delta t} \boldsymbol{u}_{\tau}-{ }^{t} \boldsymbol{u}_{\tau}\right)
$$

and

$$
\phi^{*}=\left\|\boldsymbol{p}_{\tau}^{*}\right\|-\mu^{t+\Delta t} p, \quad\left[\frac{\partial \phi}{\partial \boldsymbol{p}_{\tau}}\right]^{*}=\frac{\boldsymbol{p}_{\tau}^{*}}{\left\|\boldsymbol{p}_{\tau}^{*}\right\|}
$$

and $\boldsymbol{p}_{\tau, m_{0}}^{*}$ is calculated by the well-known return mapping algorithm [10, 22]. 
2.3. The basis of the solution technique. The solution of the frictional contact problem is based on the principle of virtual power to ensure the normal contact condition with penalty term $p_{n}=-c_{n} d$

$$
\begin{aligned}
& \sum_{\alpha}\left\{\int_{V^{\alpha}} \delta \dot{\boldsymbol{\varepsilon}} \cdot{ }^{t+\Delta t} \boldsymbol{\sigma} \mathrm{d} V-\int_{V^{\alpha}} \delta \dot{\boldsymbol{u}} \cdot{ }^{t+\Delta t} \boldsymbol{b} \mathrm{d} V-\int_{S_{p}^{\alpha}} \delta \dot{\boldsymbol{u}} \cdot{ }^{t+\Delta t} \tilde{\boldsymbol{p}} \mathrm{d} S\right\}- \\
& \quad-\int_{t+\Delta t S_{c}} \delta\left(\dot{u}_{n}^{2}-\dot{u}_{n}^{1}\right)\left(-c_{n} d\left({ }^{t+\Delta t} \boldsymbol{u}\right)\right) \mathrm{d} S-\int_{t+\Delta{ }^{t} S_{c}} \delta\left(\dot{\boldsymbol{u}}_{\tau}^{2}-\dot{\boldsymbol{u}}_{\tau}^{1}\right) \cdot{ }^{t+\Delta t} \boldsymbol{p}_{\tau} \mathrm{d} S=0 .
\end{aligned}
$$

Using the $\int_{t}^{t+\Delta t} \dot{\boldsymbol{X}} \mathrm{d} \tilde{t}=\Delta \boldsymbol{X}$ notation where $t$ denotes the dimensionless loading parameter for integration of 2.20 gives the following variational equation

$$
\begin{gathered}
\sum_{\alpha}\left\{\int_{V^{\alpha}}(\delta \Delta \varepsilon) \cdot{ }^{t+\Delta t} \boldsymbol{\sigma} \mathrm{d} V-\int_{V^{\alpha}}(\delta \Delta \boldsymbol{u}) \cdot{ }^{t+\Delta t} \boldsymbol{b} \mathrm{d} V-\int_{S_{p}^{\alpha}}(\delta \Delta \boldsymbol{u}) \cdot{ }^{t+\Delta t} \tilde{\boldsymbol{p}} \mathrm{d} S\right\}- \\
-\int_{{ }^{t+\Delta t} S_{c}} \delta\left(\Delta u_{n}^{2}-\Delta u_{n}^{1}\right)\left(-c_{n} d\left({ }^{t+\Delta t} \boldsymbol{u}\right)\right) \mathrm{d} S- \\
-\int_{t+\Delta t S_{c}} \delta\left(\Delta \boldsymbol{u}_{\tau}^{2}-\Delta \boldsymbol{u}_{\tau}^{1}\right) \cdot{ }^{t+\Delta t} \boldsymbol{p}_{\tau} \mathrm{d} S=0 .
\end{gathered}
$$

Applying the partial derivation and integral theorem for the variation equation 2.21, the following formula is written for the normal stress in the contact zone $S_{c}$,

$$
{ }^{t+\Delta t} \sigma_{n}^{1}={ }^{t+\Delta t} \sigma_{n}^{2}=c_{n} d\left({ }^{t+\Delta t} \boldsymbol{u}\right)
$$

and for the tangential stress

$$
{ }^{t+\Delta t} \boldsymbol{p}_{\tau}={ }^{t+\Delta t} p_{\tau} \boldsymbol{e}_{\tau}, \quad{ }^{t+\Delta t} p_{\tau}=\boldsymbol{e}_{\tau}{ }^{t+\Delta t} \boldsymbol{\sigma}^{1} \cdot \boldsymbol{n}^{1}=-\boldsymbol{e}_{\tau}{ }^{t+\Delta t} \boldsymbol{\sigma}^{2} \cdot \boldsymbol{n}^{2} .
$$

Of course, the tangential stress ${ }^{t+\Delta t} \boldsymbol{p}_{\tau}$ must satisfy the conditions 2.13) and 2.14, too.

2.4. Decomposition of mechanical quantities. Assuming small time steps, the mechanical quantities are decomposed into known values at time $t$ and their increments

$$
\begin{aligned}
{ }^{t+\Delta t} \boldsymbol{\sigma} & ={ }^{t} \boldsymbol{\sigma}+\Delta \boldsymbol{\sigma}, \quad{ }^{t+\Delta t} \boldsymbol{b}={ }^{t} \boldsymbol{b}+\Delta \boldsymbol{b}, \quad{ }^{t+\Delta t} p_{n}={ }^{t} p_{n}+\Delta p_{n}, \\
{ }^{t+\Delta t} \boldsymbol{u} & ={ }^{t} \boldsymbol{u}+\Delta \boldsymbol{u}, \quad{ }^{t+\Delta t} \tilde{\boldsymbol{p}}={ }^{t} \tilde{\boldsymbol{p}}+\Delta \tilde{\boldsymbol{p}}, \quad{ }^{t+\Delta t} \boldsymbol{p}_{\tau}={ }^{t} \boldsymbol{p}_{\tau}+\Delta \boldsymbol{p}_{\tau} \\
d\left({ }^{t+\Delta t} \boldsymbol{u}\right) & ={ }^{t+\Delta t}\left(u_{n}^{2}-u_{n}^{1}+h\right)={ }^{t}\left(u_{n}^{2}-u_{n}^{1}+h\right)+\Delta u_{n}^{2}-\Delta u_{n}^{1}+\Delta h \equiv \\
& \equiv{ }^{t} d+\Delta d .
\end{aligned}
$$


Subtracting the virtual powers - written in $t$ and $t+\Delta t$ time - from each other the basic functional for numerical calculations is

$$
\begin{gathered}
\sum_{\alpha}\left\{\int_{V^{\alpha}}(\delta \Delta \boldsymbol{\varepsilon}) \cdots \Delta \boldsymbol{\sigma} \mathrm{d} V-\int_{V^{\alpha}}(\delta \Delta \boldsymbol{u}) \cdot \Delta \boldsymbol{b} \mathrm{d} V-\int_{S_{p}^{\alpha}}(\delta \Delta \boldsymbol{u}) \cdot \Delta \tilde{\boldsymbol{p}} \mathrm{d} S\right\}- \\
+\int_{t+\Delta t S_{c}} \delta\left(\Delta u_{n}^{2}-\Delta u_{n}^{1}\right)\left(-c_{n}\left({ }^{t} d+\Delta u_{n}^{2}-\Delta u_{n}^{1}+\Delta h\right)\right) \mathrm{d} S- \\
-\int_{t+\Delta{ }^{t} S_{c}} \delta\left(\Delta \boldsymbol{u}_{\tau}^{2}-\Delta \boldsymbol{u}_{\tau}^{1}\right) \cdot \Delta \boldsymbol{p}_{\tau} \mathrm{d} S=0,
\end{gathered}
$$

where $\Delta \boldsymbol{p}_{\tau}$ must be determined in different ways on adhesion and on slip subregions:

- in the adhesion subregion

$$
\Delta \boldsymbol{p}_{\tau}=c_{\tau}\left(\Delta \boldsymbol{u}_{\tau}^{2}-\Delta \boldsymbol{u}_{\tau}^{1}\right)=c_{\tau} \Delta \boldsymbol{u}_{\tau}
$$

i.e., the bodies are connected by distributed elastic springs with coefficient $c_{\tau}$.

- in the slip subregion

$$
\Delta \boldsymbol{p}_{\tau}=\mu^{t+\Delta t} p_{n} \frac{\Delta \boldsymbol{u}_{\tau}}{\left\|\Delta \boldsymbol{u}_{\tau}\right\|}-{ }^{t} \boldsymbol{p}_{\tau}=\mu^{t+\Delta t} p_{n} \frac{\boldsymbol{p}_{\tau}^{*}}{\left\|\boldsymbol{p}_{\tau}^{*}\right\|}-{ }^{t} \boldsymbol{p}_{\tau}
$$

i.e., the bodies are loaded by tangential traction which computed according to the Coulomb friction.

\section{FE DISCRETIZATION WITH APPROXIMATED INCREMENTS}

The increments in displacements are approximated in the usual form

$$
\Delta \mathbf{u}^{\alpha}=\mathbf{N}^{\alpha} \Delta \mathbf{q}^{\alpha}, \quad \alpha=1,2
$$

where the shape functions in matrix $\mathbf{N}^{\alpha}$ consists of nodal modes, side modes and internal modes, $\Delta \mathbf{q}^{\alpha}$ is the vector of increments in displacement parameters [14. Increments of strains

$$
\Delta \varepsilon^{\alpha} \rightarrow \Delta \underline{\varepsilon}^{\alpha}=\mathbf{B}^{\alpha} \Delta \mathbf{q}^{\alpha}
$$

and stresses

$$
\Delta \boldsymbol{\sigma}^{\alpha} \rightarrow \Delta \underline{\boldsymbol{\sigma}}^{\alpha}=\mathbf{D}^{\alpha} \mathbf{B}^{\alpha} \Delta \mathbf{q}^{\alpha}
$$

are calculated in the usual way. Here $\mathbf{D}^{\alpha}$ is the constitutive, and $\mathbf{B}^{\alpha}$ is the straindisplacement matrix. Normal and tangential displacements in the contact surface are approximated as follows

$$
\Delta u_{n}^{\alpha}=\mathbf{L}^{\alpha} \Delta \mathbf{q}^{\alpha}, \quad \Delta \mathbf{u}_{\tau}^{\alpha}=\mathbf{L}_{\tau}^{\alpha} \Delta \mathbf{q}^{\alpha} .
$$

where $\mathbf{L}^{\alpha}$ and $\mathbf{L}_{\tau}^{\alpha}$ are contains only the localized shape functions of matrix $\mathbf{N}^{\alpha}$ for the contact domain as follows

$$
\mathbf{L}^{\alpha}=\mathbf{n}^{\alpha T} \mathbf{N}^{\alpha}(\xi, \eta, \zeta=1), \quad \mathbf{L}_{\tau}^{\alpha}=\mathbf{P}^{\alpha} \mathbf{N}^{\alpha}(\xi, \eta, q, \zeta=1),
$$


using the normal $\boldsymbol{n}^{\alpha}$ and the tangential $\boldsymbol{t}_{\xi}^{\alpha}, \boldsymbol{t}_{\eta}^{\alpha}$ vectors of contact domain.

$$
\boldsymbol{n}^{\alpha} \rightarrow \mathbf{n}^{\alpha}, \quad \boldsymbol{t}_{\xi}^{\alpha}=\frac{\partial \boldsymbol{r}^{\alpha}}{\partial \xi}, \boldsymbol{t}_{\eta}^{\alpha}=\frac{\partial \boldsymbol{r}^{\alpha}}{\partial \eta} \rightarrow \mathbf{P}^{\alpha} .
$$

The vector of total displacement parameters is ${ }^{t+\Delta t} \mathbf{q}^{\alpha}={ }^{t} \mathbf{q}^{\alpha}+\Delta \mathbf{q}^{\alpha}$.

Substituting these vectors into equation 2.25 the task is to solve the following system of equations

$$
\begin{array}{r}
\sum_{\alpha}\left\{\delta \Delta \mathbf{q}^{e T}[\underbrace{\int_{V^{\alpha}} \mathbf{B}^{T} \mathbf{D B} \mathrm{d} V \Delta \mathbf{q}^{\alpha}}_{\mathbf{K}^{\alpha}}-\underbrace{\left.\left.\int_{V^{\alpha}} \mathbf{N}^{T} \Delta \mathbf{b} \mathrm{d} V-\int_{S^{\alpha}} \mathbf{N}^{T} \Delta \tilde{\mathbf{p}} \mathrm{d} S\right]\right\}}_{\Delta \mathbf{f}_{E}^{\alpha}}\right. \\
+\delta \Delta \mathbf{q}^{T}\left[\mathbf{C}_{n}+\mathbf{C}_{\tau}\right] \Delta \mathbf{q}-\delta \Delta \mathbf{q}^{T} \mathbf{f}_{c}=0
\end{array}
$$

in which the contact stiffness matrices are

$$
\mathbf{C}_{n}=\int_{t+\Delta t S_{c}}\left[\begin{array}{c}
-\mathbf{L}^{1 T} \\
\mathbf{L}^{2 T}
\end{array}\right] c_{n}\left[\begin{array}{ll}
-\mathbf{L}^{1} & \mathbf{L}^{2}
\end{array}\right] \mathrm{d} S, \mathbf{C}_{\tau}=\int_{t+\Delta t S_{c}^{(a d h)}}\left[\begin{array}{c}
-\mathbf{L}_{\tau}^{1 T} \\
\mathbf{L}_{\tau}^{2 T}
\end{array}\right] c_{\tau}\left[\begin{array}{ll}
-\mathbf{L}_{\tau}^{1} & \mathbf{L}_{\tau}^{2}
\end{array}\right] \mathrm{d} S
$$

Introducing the notations for the quasi-triangular stiffness matrix $\mathbf{K}$

$$
\mathbf{K}=\left[\begin{array}{cc}
\mathbf{K}^{1} & \mathbf{1} \\
\mathbf{0} & \mathbf{K}^{2}
\end{array}\right]
$$

and vector of load on the contact surface

$$
\begin{array}{r}
\mathbf{f}_{c}^{(k-1)}=\int_{{ }^{t+\Delta t} S_{c}}\left[\begin{array}{c}
-\mathbf{L}^{1 T} \\
\mathbf{L}^{2 T}
\end{array}\right]\left(-c_{n} \Delta h\right) \mathrm{d} S-\int_{t+\Delta t S_{c}}\left[\begin{array}{c}
-\mathbf{L}^{1 T} \\
\mathbf{L}^{2 T}
\end{array}\right] c_{n}{ }^{t} d \mathrm{~d} S+ \\
\int_{{ }^{t+\Delta t} S_{c}^{(s l i p)}}\left[\begin{array}{c}
-\mathbf{L}_{\tau}^{1 T} \\
\mathbf{L}_{\tau}^{2 T}
\end{array}\right]\left(\mu^{t+\Delta t} p_{n}^{(k-1)} \frac{\mathbf{p}_{\tau}^{*}}{\left|\mathbf{p}_{\tau}^{*}\right|}-{ }^{t} \mathbf{p}_{\tau}\right) \mathrm{d} S,
\end{array}
$$

the final equation is written as

$$
\left[\mathbf{K}+\mathbf{C}_{n}+\mathbf{C}_{\tau}\right]^{(k)} \Delta \mathbf{q}^{(k)}=\Delta \mathbf{f}_{E}+\mathbf{f}_{c}^{(k-1)}
$$

which is solved by iterational technique. The solution process is controlled by the change in the adhesion/slip subdomains iteratively until the constraints in equations 2.10)-2.17) are satisfied on the integration points of the contact domain. It means that on all contact elements the local condition of stick and slip is fullfilled.

\section{INTERPolation With CLOSED SPLiNe}

In the present investigation it is supposed that the border curve of the resulting contact domain is a closed, smooth and continuous one. Thus it can be interpolated by a parametric spline curve. In geometric modeling and mechanical design parametric curves like B-spline curves and their rational generalization, i.e. Non-Uniform 
Rational B-spline curves or shortly NURBS, are frequently used because of their capability for defining new object, or describing existing curves and borders. They also have a usefull property, that is the ease of changeability.

To define a NURBS a simple data structure must be given in advance, which consists of three parts: the control points $\boldsymbol{v}_{i}$, the vector of knot values $\boldsymbol{u}$ and the weight factors $w_{i}$ which are associated with the control points. When one deals with non-rational B-splines the last mentioned weights are missing. Hereby a NURBS can be modified through one of the three above mentioned structure elements.

In this section only a short summary is provided about the interpolation with closed NURBS, more details can be found in the book of Piegl and Tiller [18].

A NURBS curve is defined so that

$$
\boldsymbol{c}_{r}(t)=\sum_{i=1}^{n} R_{i}^{q}(t) \boldsymbol{v}_{i}
$$

in which

$$
R_{i}^{q}(t)=\frac{N_{i}^{q}(t) w_{i}}{\sum_{j=1}^{n} N_{j}^{q}(t) w_{j}}
$$

where $n$ is the number of sample points, vector $\boldsymbol{v}_{i}$ stores the coordinates of the control point, $w_{i}$ denotes the weight factor of the $i^{t h}$ control point, $N_{i}^{q}$ is the $i^{t h}$ normalized B-spline basis function of order $q$ (degree $q-1$ ), and $\boldsymbol{c}_{r}(t)$ gives the position of a point on the curve. The basis functions are obtained through a knot vector, which defines the functions' break positions.

Consider the problem of closed NURBS interpolation to a sequence of points $\boldsymbol{d}_{i}$ $(i=1, \ldots, m)$ of a closed polygon, where $(q-1)$ constraints are additionally imposed to satisfy $C^{(q-2)}$ continuity at the endpoints of a resulting curve. It is an assumption that no two consecutive vertices on the polygon are the same.

When a cyclic knot vector [23] is applied, the required constraints are the following

$$
\boldsymbol{v}_{\lceil i\rceil}= \begin{cases}\boldsymbol{v}_{i} & \text { if } 1 \leq i \leq m, \\ \boldsymbol{v}_{i \bmod (m+1)+1} & \text { if } m<i \leq m+q-1,\end{cases}
$$

in which the operation $(i \bmod j)$ gives the residuum of the quotient $\frac{i}{j}$. This results in overlapped control points, i.e. $\boldsymbol{v}_{1}=\boldsymbol{v}_{m+1}, \ldots, \boldsymbol{v}_{q-1}=\boldsymbol{v}_{m+q-1}$.

To determine the control points the following linear equation must be solved

$$
\boldsymbol{d}_{i}=\boldsymbol{c}_{r}\left(t_{i}\right)=\sum_{k=1}^{n} R_{k}^{q}\left(t_{i}\right) \boldsymbol{v}_{\lceil k\rceil} \quad 1 \leq i \leq m .
$$

Rewriting equation 4.4 in matrix form using equations 4.2 and 4.3 leads to

$$
\mathbf{N}^{q} \mathbf{V}=\mathbf{D}
$$


in which

$$
\mathbf{N}^{q}=\frac{1}{\sum_{j=1}^{n} N_{j}^{q}\left(t_{i}\right) w_{j}}\left[\begin{array}{ccc}
w_{1} N_{1}^{q}\left(t_{1}\right)+w_{m+1} N_{m+1}^{q}\left(t_{1}\right) & \ldots & w_{m} N_{m}^{q}\left(t_{1}\right) \\
w_{1} N_{1}^{q}\left(t_{2}\right)+w_{m+1} N_{m+1}^{q}\left(t_{2}\right) & \ldots & w_{m} N_{m}^{q}\left(t_{2}\right) \\
\vdots & & \\
w_{1} N_{1}^{q}\left(t_{m}\right)+w_{m+1} N_{m+1}^{q}\left(t_{m}\right) & \ldots & w_{m} N_{m}^{q}\left(t_{m}\right)
\end{array}\right]
$$

where it is required to make summation in the first few $(q-1)$ columns because of the overlapped control points and

$$
\mathbf{V}=\left[\begin{array}{ccc}
v_{1 x} & v_{1 y} & v_{1 z} \\
v_{2 x} & v_{2 y} & v_{2 z} \\
\vdots & & \\
v_{m x} & v_{m y} & v_{m z}
\end{array}\right], \quad \mathbf{D}=\left[\begin{array}{ccc}
d_{1 x} & d_{1 y} & d_{1 z} \\
d_{2 x} & d_{2 y} & d_{2 z} \\
\vdots & & \\
d_{m x} & d_{m y} & d_{m z}
\end{array}\right] .
$$

Finally, a square coefficient matrix $\mathbf{N}^{q}$ with size $m \times m$ is obtained. Thus the $m$ interpolation points determine $n$ control points since the $m+1^{t h}, m+2^{t h}, \ldots$, control points are the same as the $1^{\text {st }}, 2^{\text {nd }}, \ldots$ control points.

4.1. Choosing the parameters. The first step during interpolation is to assign parameters to different interpolation points, which are on the resulting parametric curve. It means that the parameters $t_{i}(i=1, \ldots, m)$ are chosen in a way that the following equation holds true

$$
\boldsymbol{d}_{i}=\boldsymbol{c}_{r}\left(t_{i}\right) \quad 1 \leq i \leq m .
$$

In Figure 3 the curve must pass over five given interpolation points, i.e. five parameters $t_{1}, \ldots, t_{5}$ are needed to be chosen in the first step.

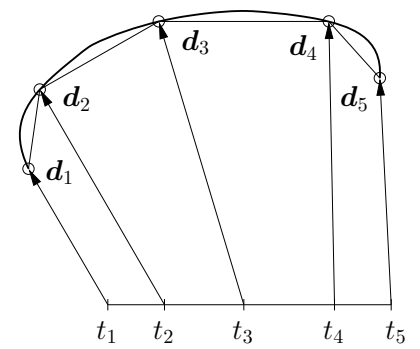

Figure 3. Relationship between the interpolation points and the parameters

Several techniques exist for making this assignment between the interpolation points and the parameters, see Refs. [18, 19, 23]. In this paper the parameters are chosen according to the chord length.

If the interpolating curve is closed, i.e. $\boldsymbol{d}_{1}=\boldsymbol{d}_{m+1}$, the polygon - given by $\boldsymbol{d}_{i}$ $(i=1, \ldots, m)$ different interpolation points - is also closed. The parameters are 
chosen by chord length with the following assumptions: Parameter $t_{1}=0$ is assigned to $\boldsymbol{d}_{1}$, while parameter $t_{m+1}=1$ is assigned to the imagined point $\boldsymbol{d}_{m+1}$.

Other parameters $t_{i}(i=2, \ldots, m)$ can be computed with the help of chord lengths

$$
\begin{aligned}
t_{1} & =0, \quad t_{m+1}=1, \\
t_{i} & =\frac{1}{L_{c}} \sum_{j=2}^{i}\left|\boldsymbol{d}_{j}-\boldsymbol{d}_{j-1}\right| \quad 1<i<m+1,
\end{aligned}
$$

where $L_{c}$ is the length of the closed polygon

$$
L_{c}=\sum_{i=2}^{m+1}\left|\boldsymbol{d}_{i}-\boldsymbol{d}_{i-1}\right| .
$$

4.2. Generating the knot vector. After choosing the parameters, the next important step for creating an interpolation curve is to generate the knot vector $\boldsymbol{u}=$ $\left[u_{1}, \ldots, u_{l}\right]$ which is needed to obtain the normalized B-spline basis functions.

Since the knot vector is searched for a closed interpolating curve the parameters $t_{1}, t_{2}, \ldots, t_{m}$ are known. The number of control points which can be calculated from equation 4.5 is $n=m+q-1$, therefore the number of knot values, which are written in the knot vector $\boldsymbol{u}$, is $l=m+2 q-1$, see. 23 .

In this way there exist no such a knot value which has $k>1$ multiplicity. For generating the knot values the following term is applied, which is partially based on Ref. 23

$$
\begin{aligned}
u_{-k} & =u_{1-k}+u_{n-q-k+2}-u_{n-q-k+3} \quad k=1, \ldots, q-1, \\
u_{1} & =0, \quad u_{n-q+2}=1 \\
u_{i} & =\frac{1}{2 z+1} \sum_{j=i-z}^{i+z} s_{j} \quad i=2, \ldots, n-q+1, \\
u_{n-q+2+k} & =u_{n-q+1+k}+u_{k+1}-u_{k} \quad k=1, \ldots, q-1
\end{aligned}
$$

in which $z=\left\lfloor\frac{q-1}{2}\right\rfloor,\lfloor x\rfloor$ means the greatest integer which is not greater than $x$. For calculating $s_{j}(j=-(z+2), \ldots, m+z)$ refer to the following rules

$$
s_{j}= \begin{cases}u_{1}+t_{m+1+j}-t_{m+1} & \text { if }-(z-1) \leq j \leq-1, \\ t_{j} & \text { if } 1 \leq j \leq(m+1), \\ u_{n-q+2}+t_{j-m-1} & \text { if }(m+2) \leq j \leq(m+z)\end{cases}
$$

where $u_{n-q+2}=t_{m+1}=1$.

For better understanding of the above formulation let us see a numerical example to generate the knots for a fourth order $(q=4)$ interpolation spline. There are $m=8$ interpolation points given, and the aim is to create a closed spline which crosses all the interpolation points $\boldsymbol{d}_{1}, \ldots, \boldsymbol{d}_{8}$. The parameters $t_{1}, \ldots, t_{8}$ are also calculated, according to the chord length, and listed in Table 1 . 
Table 1. Parameters for $(m=8)$ interpolation points

\begin{tabular}{ccccccccc}
$t_{1}$ & $t_{2}$ & $t_{3}$ & $t_{4}$ & $t_{5}$ & $t_{6}$ & $t_{7}$ & $t_{8}$ & $t_{9}$ \\
\hline 0 & $\frac{1}{8}$ & $\frac{2}{8}$ & $\frac{3}{8}$ & $\frac{4}{8}$ & $\frac{5}{8}$ & $\frac{6}{8}$ & $\frac{7}{8}$ & 1
\end{tabular}

The task is to generate the cyclic knot vector $\boldsymbol{u}$. The number of control points is $n=m+q-1=11$, therefore the number of knot values is $l=n+q=15$. The parameters for the cyclic knot vector $\boldsymbol{u}$ are $u_{1}=0$ and $u_{n-q+2}=u_{9}=1$ while the rest can be obtained by means of equations (4.11) and (4.12). The results are summarised in Table 2 .

Table 2. Cyclic knot vector $(q=4, m=8)$

\begin{tabular}{ccccccccccccccc}
$(1)$ & $(2)$ & $(3)$ & $(4)$ & $(5)$ & $(6)$ & $(7)$ & $(8)$ & $(9)$ & $(10)$ & $(11)$ & $(12)$ & $(13)$ & $(14)$ & $(15)$ \\
$u_{-3}$ & $u_{-2}$ & $u_{-1}$ & $u_{1}$ & $u_{2}$ & $u_{3}$ & $u_{4}$ & $u_{5}$ & $u_{6}$ & $u_{7}$ & $u_{8}$ & $u_{9}$ & $u_{10}$ & $u_{11}$ & $u_{12}$ \\
\hline$-\frac{3}{8}$ & $-\frac{2}{8}$ & $-\frac{1}{8}$ & 0 & $\frac{1}{8}$ & $\frac{2}{8}$ & $\frac{3}{8}$ & $\frac{4}{8}$ & $\frac{5}{8}$ & $\frac{6}{8}$ & $\frac{7}{8}$ & 1 & $\frac{9}{8}$ & $\frac{10}{8}$ & $\frac{11}{8}$
\end{tabular}

4.3. Numerical example. A simple Fortran code is developed to interpolate a set of points which are chosen by the contact search algorithm, i.e. the points are all along the contact-separation border. Now let us consider a 2D interpolation across $(m=8)$ points $\boldsymbol{d}_{i},(i=1, \ldots, 8)$ which are in the same plane (see Figure 4 .).

Table 3. Coordinates of interpolation points

\begin{tabular}{rrrr|rrrr}
$\boldsymbol{d}_{i}$ & $x$ & $y$ & $z$ & $\boldsymbol{d}_{i}$ & $x$ & $y$ & $z$ \\
\hline \hline $\boldsymbol{d}_{1}$ & $-\sqrt{2}$ & $-\sqrt{2}$ & 5.0 & $\boldsymbol{d}_{5}$ & $\sqrt{2}+0.2$ & $\sqrt{2}+0.2$ & 5.0 \\
\hline $\boldsymbol{d}_{2}$ & -2.0 & 0.0 & 5.0 & $\boldsymbol{d}_{6}$ & 2.0 & 0.0 & 5.0 \\
\hline $\boldsymbol{d}_{3}$ & $-\sqrt{2}-0.2$ & $\sqrt{2}+0.2$ & 5.0 & $\boldsymbol{d}_{7}$ & $\sqrt{2}$ & $-\sqrt{2}$ & 5.0 \\
\hline $\boldsymbol{d}_{4}$ & 0.0 & 3.0 & 5.0 & $\boldsymbol{d}_{8}$ & 0.0 & -2.0 & 5.0 \\
\hline
\end{tabular}

It is obvious, that the number of control points belonging to the closed interpolation spline curve is $(n=11)$. Since the order of the B-spline basis function is chosen for $q=4$, the first and last three control points are overlapped. The calculated

Table 4. Coordinates of control points

\begin{tabular}{lrrr|rrrr}
$\boldsymbol{v}_{i}$ & $x$ & $y$ & $z$ & $\boldsymbol{v}_{i}$ & $x$ & $y$ & $z$ \\
\hline \hline $\boldsymbol{v}_{8}$ & 0.0 & -2.21321 & 5.0 & $\boldsymbol{v}_{4}$ & 0.0 & 3.69991 & 5.0 \\
\hline $\boldsymbol{v}_{1}=\boldsymbol{v}_{9}$ & -1.58342 & -1.57357 & 5.0 & $\boldsymbol{v}_{5}$ & 1.72789 & 1.75406 & 5.0 \\
\hline $\boldsymbol{v}_{2}=\boldsymbol{v}_{10}$ & -2.16753 & 0.06703 & 5.0 & $\boldsymbol{v}_{6}$ & 2.16753 & 0.06703 & 5.0 \\
\hline $\boldsymbol{v}_{3}=\boldsymbol{v}_{11}$ & -1.72789 & 1.75406 & 5.0 & $\boldsymbol{v}_{7}$ & 1.58342 & -1.57357 & 5.0
\end{tabular}




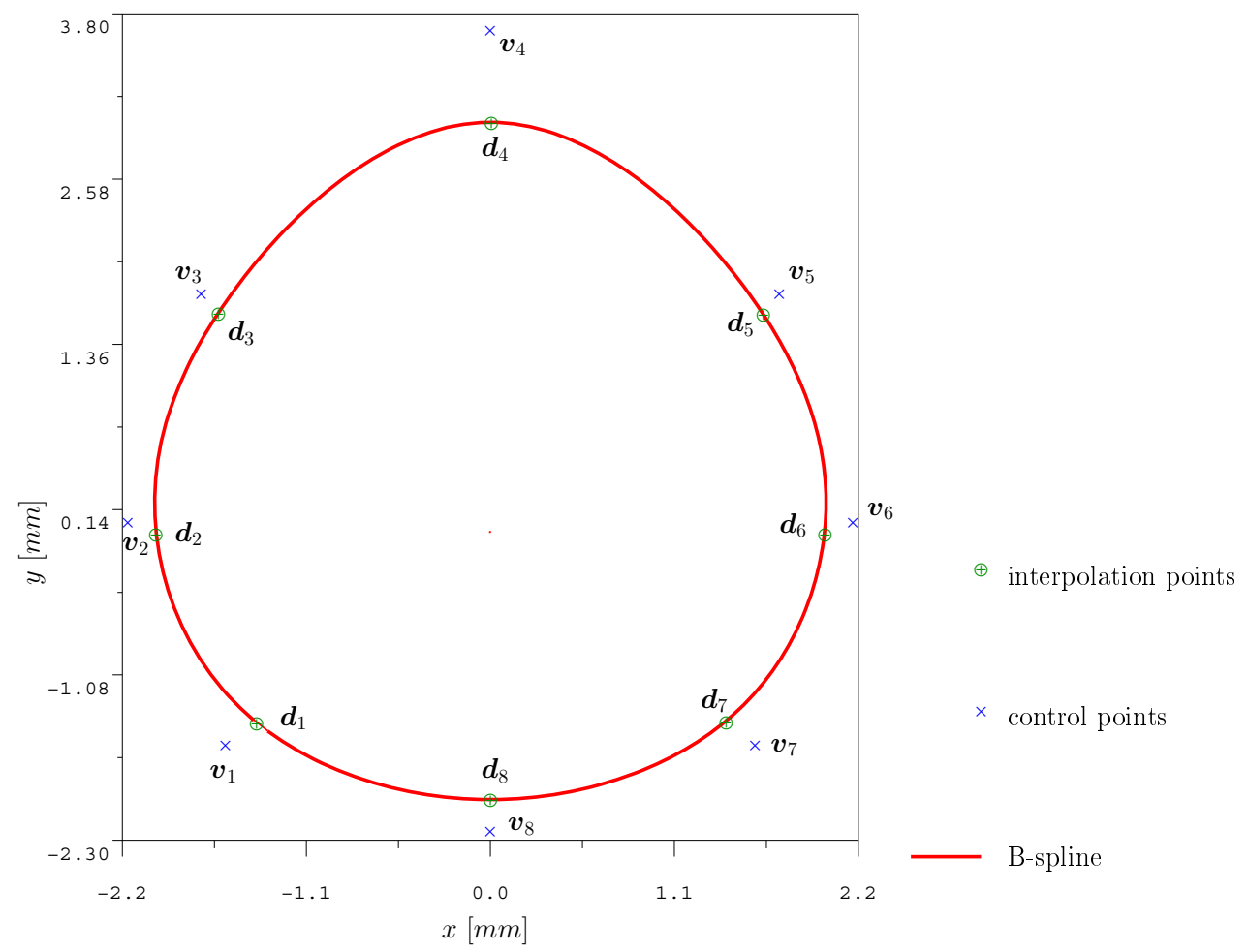

Figure 4. Interpolation with a spline curve generated numerically.

coordinates of the control points $\boldsymbol{v}_{i},(i=1, \ldots, 11)$ are illustrated in Figure 4 . For the sake of simplicity, here the weights $w_{i}$ are prescribed to 1 . The parameters for the interpolation points are generated according to chord lengths.

The resulting two-dimensional NURBS curve is drawn in Figure 4 which is generated by the calculated control points. For drawing the spline, 303 points are determined along the curve and between the points a line segments are used.

\section{EXAMPle FOR 3D CONTACT}

Here the contact of two bodies is considered (shown in Figure 5). The upper body is a rigid sphere and the lower one is an elastic block with the geometrical data $R=800 \mathrm{~mm}, a=5 \mathrm{~mm}, b=c=10 \mathrm{~mm}$. The material parameters are the modulus of elasticity $E_{1}=\infty, E_{2}=2.1 \cdot 10^{5} \mathrm{MPa}$ and the Poisson-ratio $\nu_{2}=0.3$. The contact 
problem is investigated in frictional case as well. Here three different coefficients of friction are applied for tree different computations, $\mu_{1}=0.08, \mu_{2}=0.1, \mu_{3}=0.12$. The upper body is loaded on its top by a prescribed displacement $w_{0}=5.0 \cdot 10^{-3} \mathrm{~mm}$.

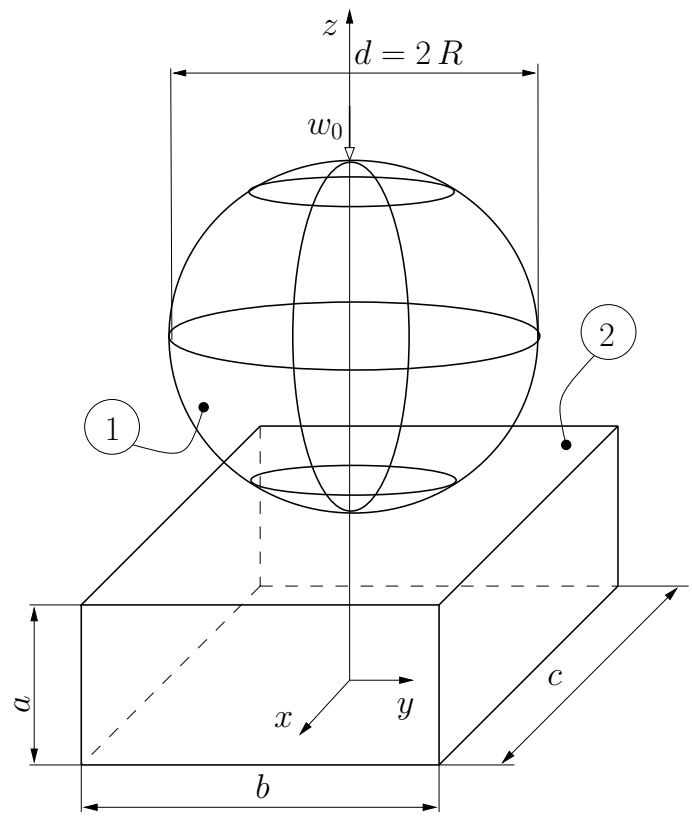

Figure 5. Geometry of the contacting bodies.

Two types of contact problems are investigated. Firstly, a normal contact problem is analysed in order to make a comparison between the solution achieved by the Hertztheory and the finite element computation. Secondly, frictional contact is examined to get to know more about the stick and slip zones with the use of different friction coefficients.

Approximating the analytical solution according to the HERTz-theory [1] the following formulae are used

$$
a_{0}=\sqrt[3]{\frac{3}{4} F k \frac{R_{1} R_{2}}{R_{1}+R_{2}}}, \quad p_{0}=\frac{\sqrt[3]{6}}{\pi} \sqrt[3]{\frac{F}{k^{2}}\left(\frac{R_{1}+R_{2}}{R_{1} R_{2}}\right)^{2}}
$$

in which $F$ is the applied compression force, $a_{0}$ is the size of the contact domain, $p_{0}$ is the maximal contact pressure, $R_{1}$ and $R_{2}$ are the radii of the upper and the lower body, respectivelly. Here the lower contacting body is a brick therefore $R_{2}=\infty$ i.e.

$$
\frac{R_{1}+R_{2}}{R_{1} R_{2}}=\frac{1}{R_{1}} \quad \text { and } \quad \frac{R_{1} R_{2}}{R_{1}+R_{2}}=R_{1},
$$


and since the upper body is rigid

$$
k=\frac{1-\nu_{2}^{2}}{E_{2}}
$$

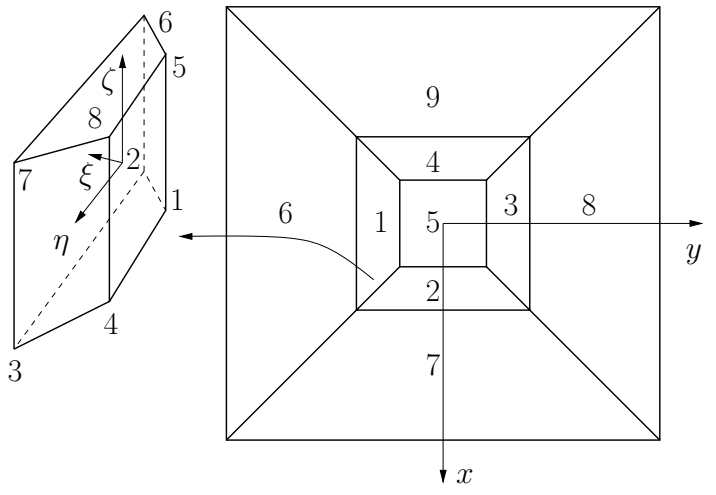

a.)

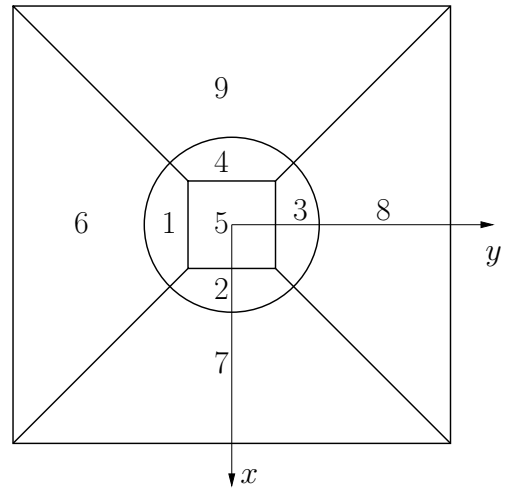

b.)

Figure 6. Applied FE meshes without friction.

In all our numerical investigations p-extension finite elements are used. The applied FE meshes are shown in Figure 6 and Figure 7 . In case of the normal contact problem the aim is to identify the border of contact zone. Therefore only 9 elements are applied. Figure 6a illustrates the mesh which was used only for test reasons and as an initial point for computations. Naturally, it cannot be expected accurate results using such a coarse mesh for a problem in which a rigid spherical intender is used. Therefore the mesh was modified according to Figure $6 \mathrm{~b}$ where the borders of elements 1, 2, 3, 4 are interpolated by properly chosen splines.

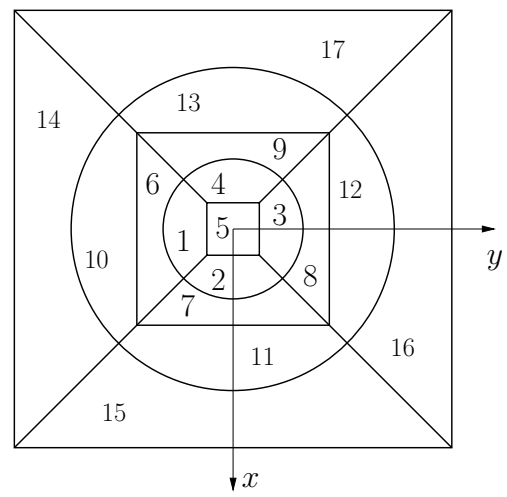

a.)

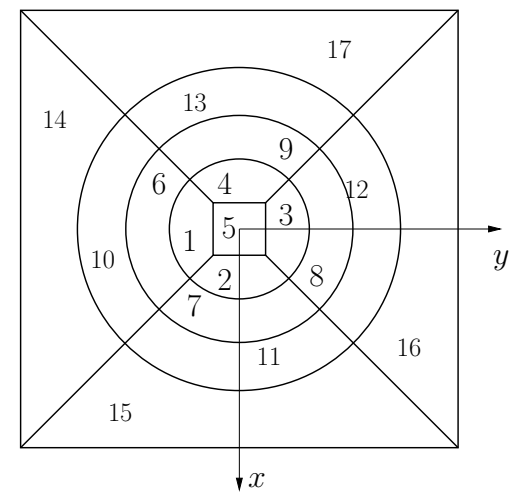

b.)

Figure 7. Applied FE meshes with friction. 
In frictional case not only the contact border but also the stick and slip zones are investigated. It means that two subdomains exist in the real contact surface and between them the algorithm must identify also a smooth well-defined border. Here more elements are used to distinguish the sticking and slipping elements and to ensure smooth stress distributions on these zones.

5.1. The search of border points. Determining the points on contact-separation or stick-slip borders is a key task before making the interpolation. Figure 8 shows the points which must be identified. The code developed uses eight different directions along which the search is performed. These directions chosen follow the edges of elements and the integration points in the middle of each contact elements.

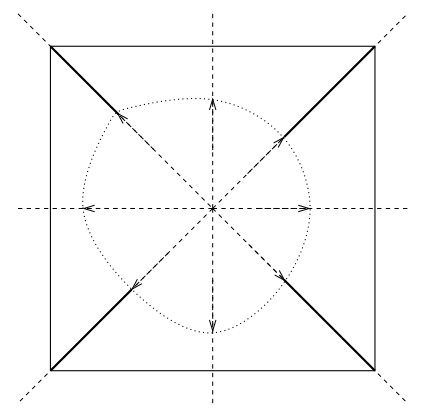

Figure 8. Directions on which the points are to be identified.

The search of border points are based on the computed results determined in previous step. In the integration points of each directions the contact pressure is produced and before the supposed border point all the pressure values have the same sign. See the illustration in Figure 9.
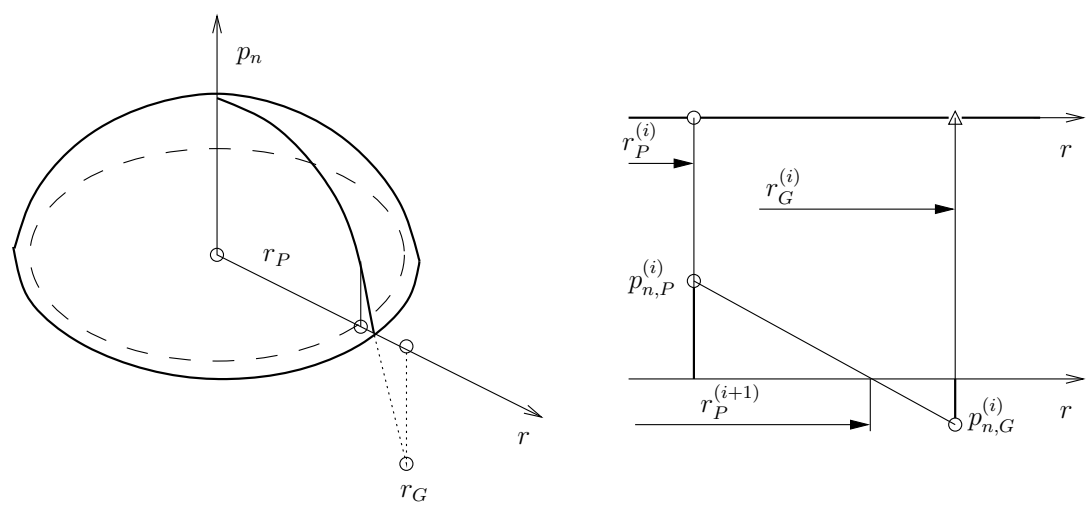

Figure 9. Linear approximation of a border point. 
The border point can be identified after some finite steps, using the following linear iteration schema

$$
r_{P}^{(i+1)}=r_{P}^{(i)}+\frac{p_{n, P}^{(i)}}{p_{n, P}^{(i)}-p_{n, G}^{(i)}}\left(r_{G}^{(i)}-r_{P}^{(i)}\right) .
$$

According to equation (5.3) the eight points of the border are determined which are interpolated with a single closed B-spline. The advantage of this procedure can be easily noticable for non-symmetrical identation problems where the resulting contact zone is a priory not known .

5.2. Results for normal contact. Two types of meshes are applied. In the first case straight bordered elements are used. The results for these computations are illustrated in Figures 10 and 11 .

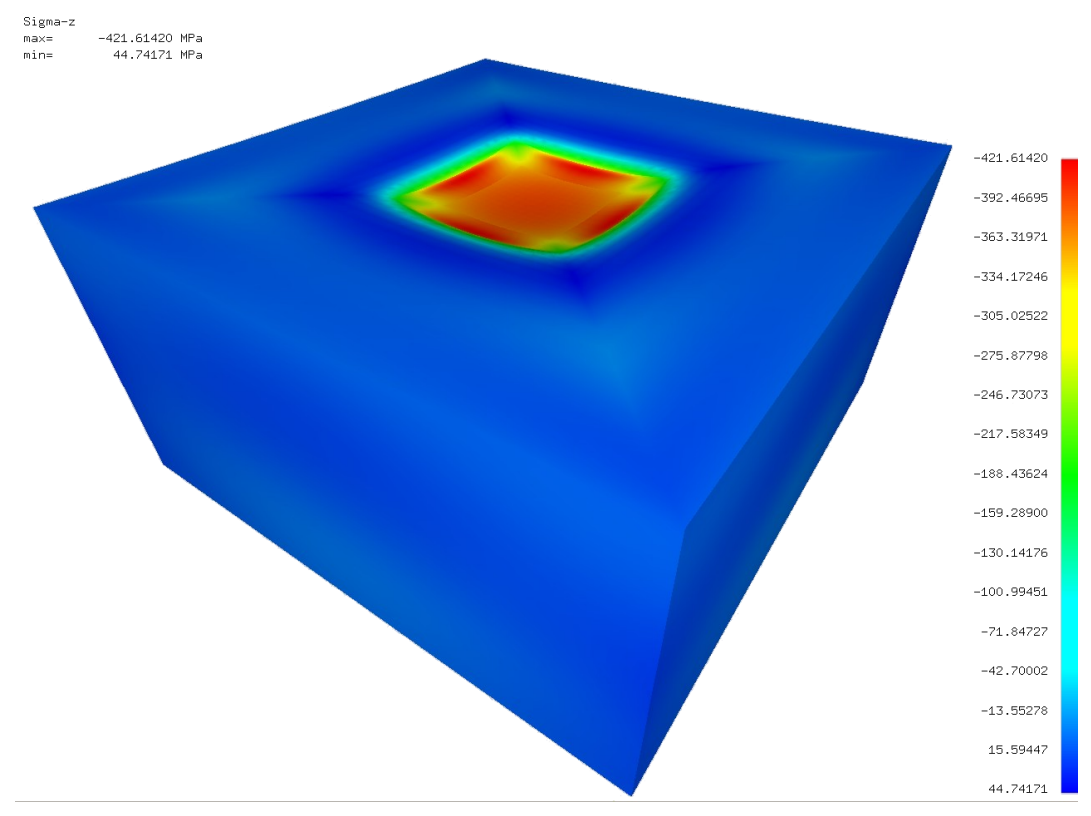

Figure 10. Oscillation in $\left(-\sigma_{z}\right)$ normal stress distribution.

On Figure 11 the normal pressure is drawn as a function of $x$ coordinates. The polynomial degree is increased from 4 to 8 . The normal pressure can be computed from the penalty term $\left(-c_{N} \cdot d^{-}\right)$and the derivatives of displacements $\left(-\sigma_{z}\right)$, respectively. Results shown in Figure 11 are not acceptable because it cannot describe the smooth pressure distribution along the axis $x$. Hence the application of straight bordered elements are not adequate.

In the second case spline bordered elements are applied. Computations show smoother pressure distribution, as can be seen in Figures 12 and 13 . This shows 


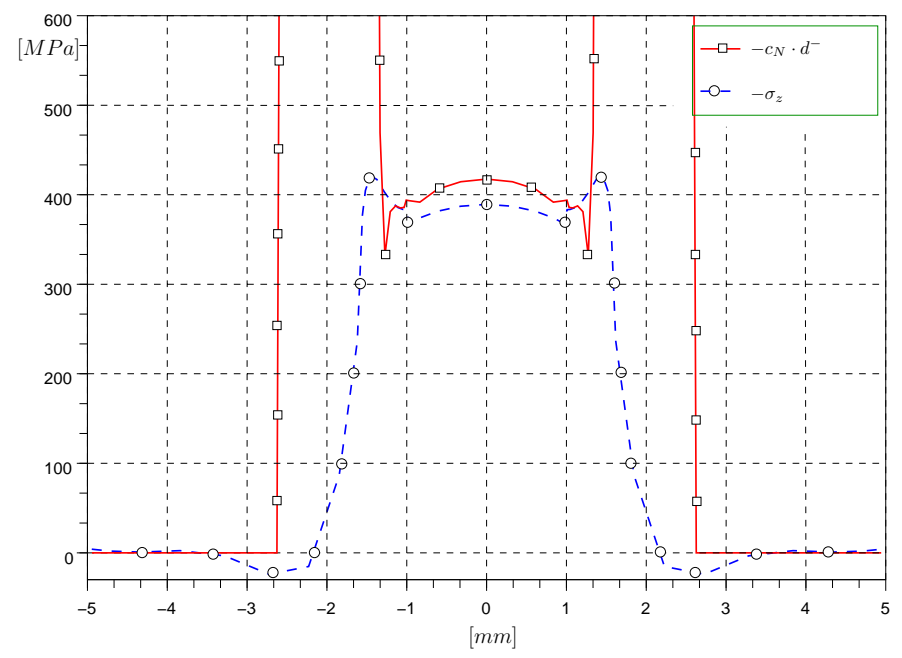

Figure 11. Contact pressure calculated by straight element borders.

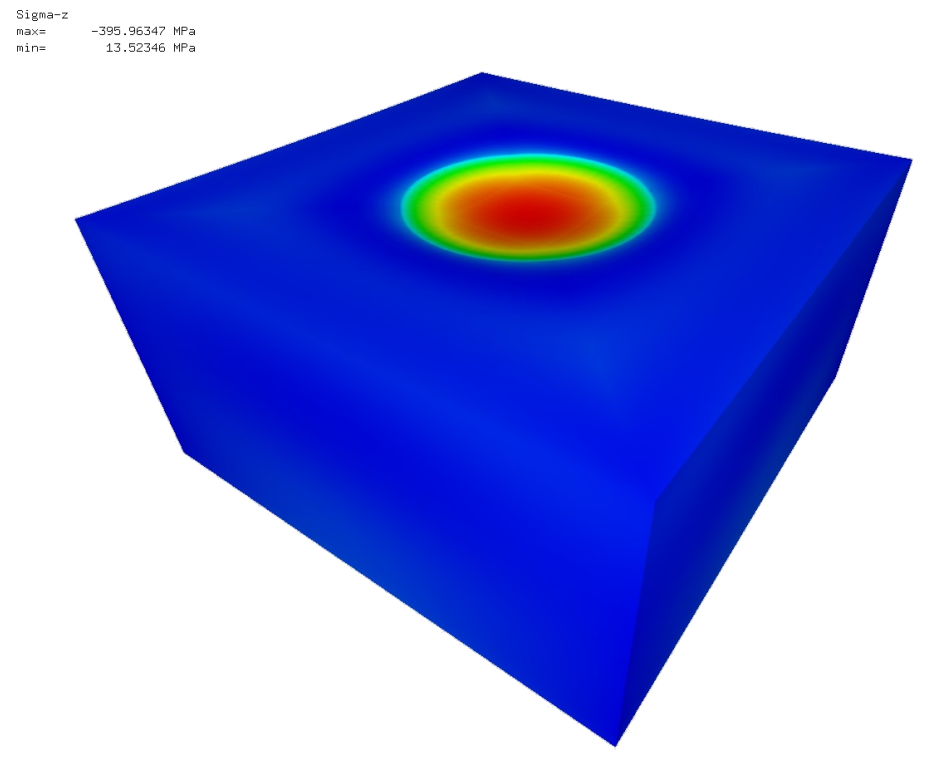

\section{$-395.96347$ $-344.77761$ -319.1846 ? $-293.59174$ $-267.99891$ $-242.40597$ $-216.81294$ $-191.22000$ $-165.62707$ $-140.03414$ $-114.44120$ $-88.84827$ $-63.25534$ $-37.66240$ $-12.06947$} 13.52346

Figure 12. Oscillation free results in case of $\left(-\sigma_{z}\right)$.

the analytical results coming from equations (5.1) and the numerical results, respectively. In numerical cases the applied polynomial degree is also varied from 4 to 8. 


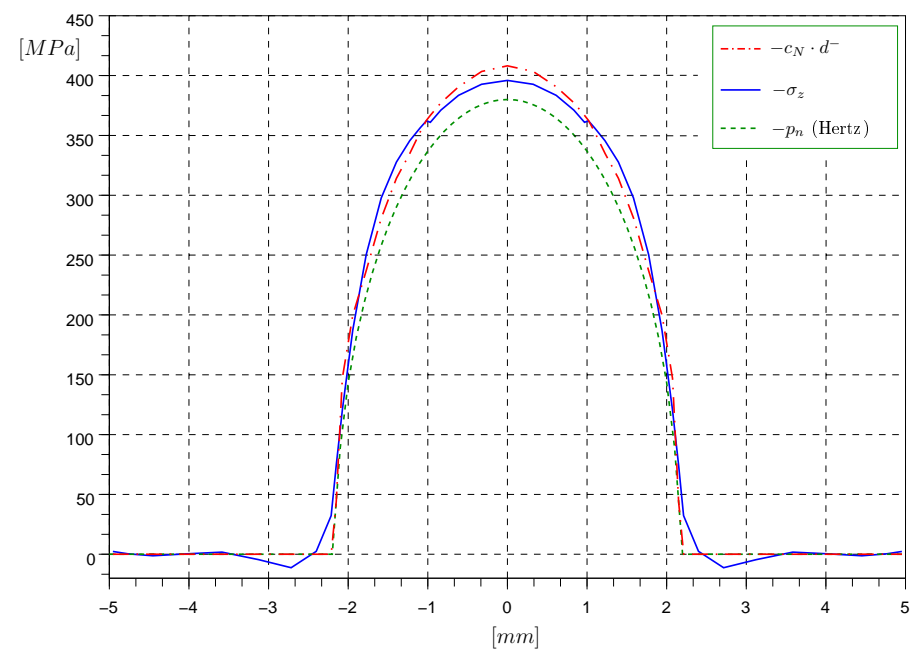

Figure 13. Contact pressure compared with the Hertzian result.

Table 5. Results of normal contact with spline border elements $w=-0.005 \mathrm{~mm}$

\begin{tabular}{rrrr} 
& $p$ & $a_{0}[\mathrm{~mm}]$ & $p_{n}[\mathrm{MPa}]$ \\
\hline HERTZ & & 2.000 & 367.28 \\
\hline \hline FEM & 4 & 2.100 & 491.76 \\
\hline & 5 & 2.111 & 462.24 \\
\hline 6 & 2.139 & 423.66 \\
\hline 7 & 2.157 & 401.34 \\
\hline & 2.161 & 395.96 \\
\hline
\end{tabular}

The discrepancy between the analytical and the numerical results - see Tab. 5 can be explained by the different assumptions used for the boundary value problem in the theoretical and numerical approaches, i.e. the lower body has finite dimensions in all directions.

If one makes a comparison between these numerical results, it will be obviously seen that the application of positioning elements [14] with spline borders is suitable. Figure 14 draws the relative error in energy norm in three different computation series: straight bordered elements with and without remeshing, and elements bounded by a positioning spline curve.

5.3. Results for frictional contact. Here, friction is taken into account. After the solution of the normal contact problem the effects of friction are also applied. Therefore it is computed with an iterational procedure. 


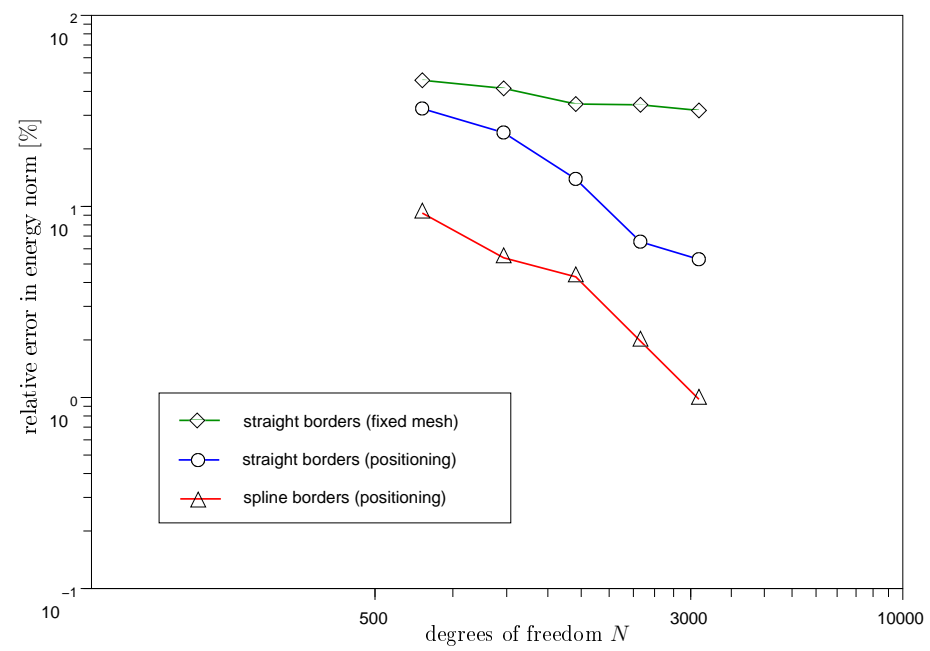

Figure 14. Relative errors without friction.

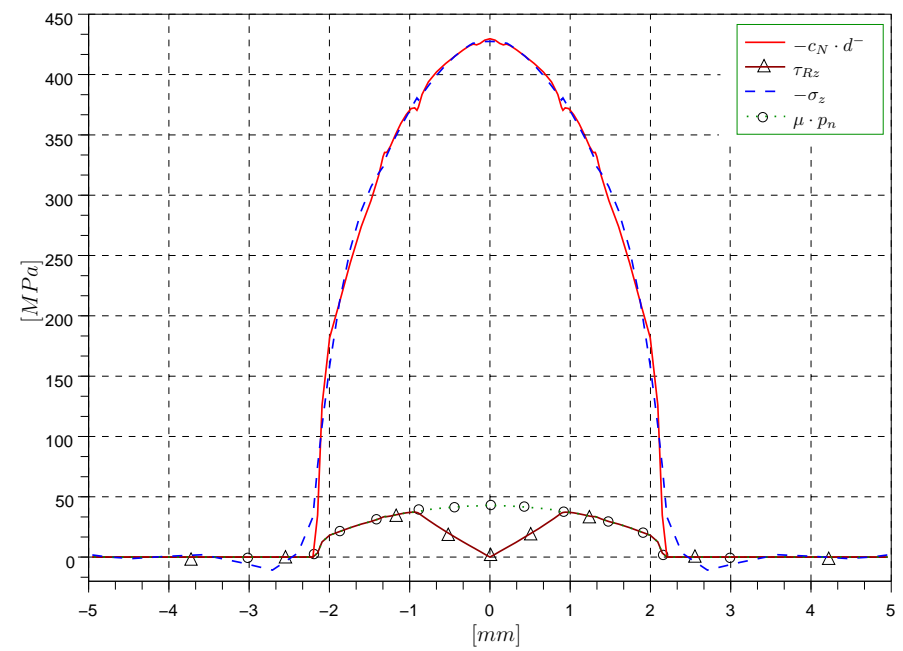

Figure 15. Contact pressure and shear stress with $\mu=0.10$.

As is known, in the case of frictional contact there are two additional domains inside the contact zone. These are the stick and slip domains which are distinguished according to the relation between shear and the normal stresses. The size of the adhesion zone is $r_{a d h}=0.96 \mathrm{~mm}$, while the slip zone is $0.96 \mathrm{~mm} \leq r_{\text {slip }} \leq 2.16 \mathrm{~mm}$ which can be identified from Figure 15. 
Results computed with $\mu=0.10$ are illustrated in Figure 15 where $\tau_{R z}$ means the shear stress.

Figure 16 shows the effects of the friction coefficient on relative energy norm. The polynomial order of shape functions is increased from 4 to 8 in order to see the convergence in the solution.

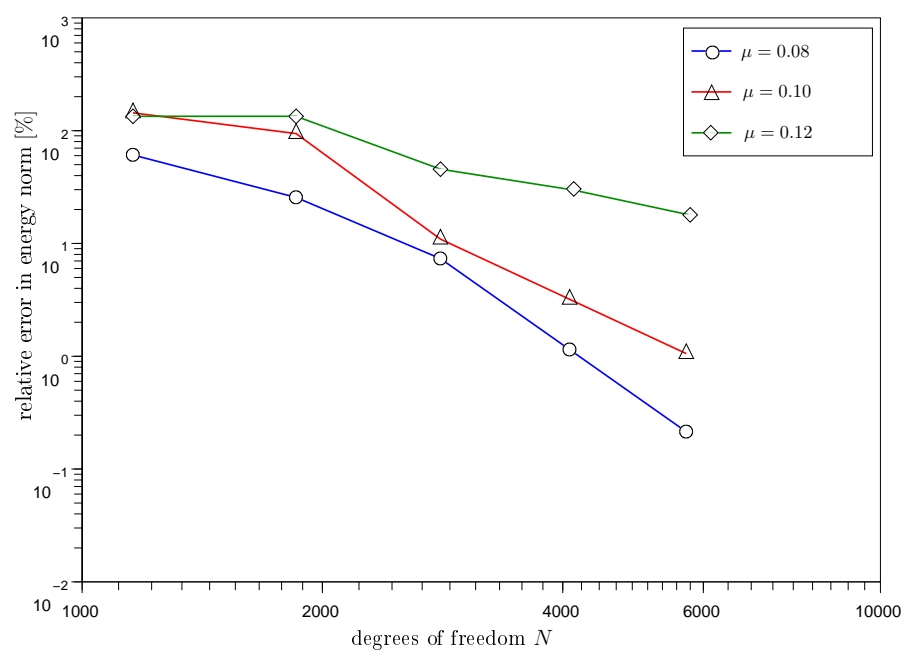

Figure 16. Relative errors with different friction coefficients.

Using the spline border positioning process the error in energy norm is smaller comparing to meshes without spline interpolation.

\section{Conclusions}

Unilateral contact problems have been solved by $p$-extension of the finite element method with the following assumptions:

- the contact region is single connected,

- the border of the contact region is a smooth curve, and the border curve of the stick and slip zones is also smooth.

In order to achieve high accuracy, the treatment of weak singularities along the border of the contact region and along the border curve of the stick and slip zones are needed. The positiong of the spline to the contact border and to the stick-and-splip zone proved to be an efficient method.

The border curves related to the contact zones are interpolated by B-splines using only a couple of design parameters. The localization of the border curve of the stick 
and slip zones requires more iteration steps than to find the border of the contact region.

Acknowledgements. This research was (partially) carried out in the framework of the Center of Excellence of Innovative Engineering Design and Technologies at the University of Miskolc.

\section{REFERENCES}

1. Hertz, H.: Über die Berührung fester elastischer Körper. Journal für die Reine und Angewandte Mathematik, 29, (1882), 156-171.

2. Signorini, A.: Sopra akune questioni di elastostatica. Ph.D. thesis, Atti della Societa Italiana per il Progresso delle Scienze, 1933.

3. Muskhelishvili, N. I.: Some Basic Problems of the Mathematical Theory of Elasticity. Moscow, 3rd edn., 1949. In Russian (English transalation by J.R.M. Radok, Noordhoff, 1953.).

4. Gallin, L. A.: Contact Problems in the Theory of Elasticity. Moscow, 1953. In Russian (English traslation by H. Moss, North Carolina State College, Dep. Math., 1961.).

5. Goldsmith, W.: Impact: The theory and physical behavior of colliding solids. London: Edward Arnold, 1960.

6. Fichera, G.: Problemi elastostatici con cincoli unilaterale: il problema di signorini con ambigue condizioni al contorno. Series 8, 7, 91.

7. Kikutchi, N. and Oden, J. T.: A study of variational inequalities and finite element methods. Contact problems in elasticity, Philadelphia: SIAM, 1988.

8. Johnson, K. L.: Contact Mechanics. Cambridge University Press, 1985.

9. Kalker, J. J.: A course of contact mechanics. TU Delft, Neatherland, 1985.

10. Wriggers, P.: Computational Contact Mechanics. John Wiley \& Sons, Ltd., 2002.

11. Turner, M. J., Clough, R. W., Martin, H. C., and Topp, L. J.: Stiffness and deflection analysis of complex structures. Journal of the Aeronautical Sciences, 23, (1956), 805-823.

12. Wilson, E. A. and Parsons, B.: Finite element analysis of elastic contact problems using differential displaments. International Journal for Numerical Methods in Engineering, 2, (1970), 387-395.

13. Szabó, B. A. and Babuška, I.: Finite Element Analysis. John Wiley \& Sons, 1991.

14. PÁczelt, I., Szabó, B., and Szabó, T.: Solution of contact problem using the $h p$ version of the finite element method. Computers and Mathematics with Applications, 38, (1999), 49-69.

15. PÁczelt, I. and Szabó, T. .: Solution of contact optimization problems of cylindrical bodies using $h p$-fem. International Journal for Numerical Methods in Engineering, 53, (2002), 123-146.

16. Düster, A., Niggl, A., NüBel, V., and Rank, E.: A numerical investigation of highorder finite elements for problems of elasto-plasticity. 17, (2002), 429-437.

17. DE Boor, C.: A Practical Guide to Splines. Applied Mathematical Sciences, SpringerVerlag, New York, 1978. 
18. Piegl, L. and Tiller, W.: The NURBS book. Berlin: Springer, 1995.

19. Beatty, R. H. B. J. C. and Barsky, B. A.: An Introduction to Splines for use in Computer Graphics \& Geometric Modeling. 1987.

20. LurJe, A. I.: Theory of Elasticity. Nauka, Moscow, 1970. In Russian.

21. Michalowski, R. and Mróz, Z.: Associated and non-associated sliding rules in contact friction problems. Archives of Mechanics, 30, (1978), 259-276.

22. Simo, J. C. and Hughes, T. J. R.: Computational Inelasticity. Springer, New York, Berlin, 1998.

23. PARK, H. and KIM, K.: Smooth surface approximation to serial cross-sections. Computer-Aided Design, 28, (1999), 995-1005. 